Title:

Microcellular Ceramic Foams for Radar Absorbing Structures

Author(s):

Jeffrey Huling, MST-4

David Phillips, MST-4

Submitted to:

DOE Office of Scientific and Technical Information (OSTI)

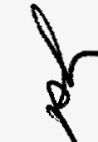

DISTRIBUTION OF THIS DOCUMENT IS UNLMIED

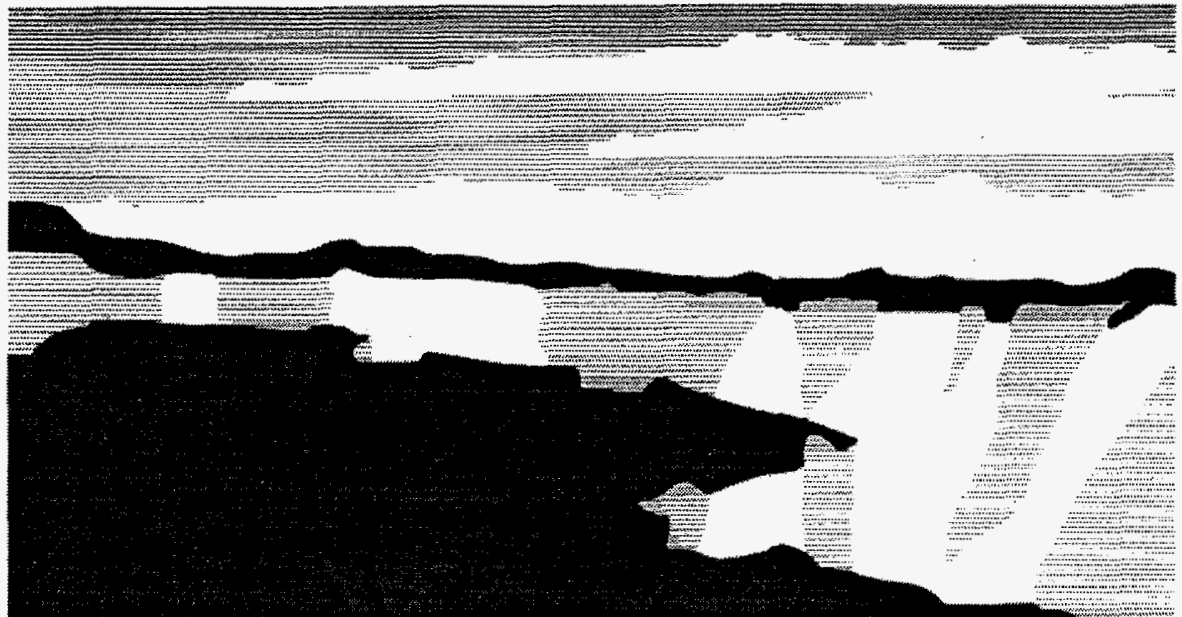

Los Alamos National Laboratory, an affirmative action/equal opportunity employer, is operated by the University of California for the U.S. Department of Energy under contract W-7405-ENG-36. By acceptance of this anticle, the publisher recognizes that the U.S. Government retains a nonexclusive, royaltyfree license to publish or reproduce the published form of this contribution, or to allow others to do so, for U.S. Government purposes. The Los Alamos National Laboratory requests that the publisher identify this article as work performed under the auspices of the U.S. Department of Energy. 


\section{DISCLAIMER}

Portions of this document may be illegible in electronic image products. Images are produced from the best available original document. 


\section{DISCLAIMER}

This report was prepared as an account of work sponsored by an agency of the United States Government. Neither the United States Government nor any agency thereof, nor any of their employees, makes any warranty, express or implied, or assumes any legal liability or responsibility for the accuracy, completeness, or usefulness of any information, apparatus, product, or process disclosed, or represents that its use would not infringe privately owned rights. Reference herein to any specific commercial product, process, or service by trade name, trademark, manufacturer, or otherwise does not necessarily constitute or imply its endorsement, recommendation, or favoring by the United States Government or any agency thereof. The views and opinions of authors expressed herein do not necessarily state or reflect those of the United States Government or any agency thereof. 


\title{
Microcellular Ceramic Foams for Radar Absorbing Structures
}

\author{
Jeffrey Huling* and David Phillips
}

\begin{abstract}
This is the final report of a one-year, Laboratory-Directed Research and Development (LDRD) project at the Los Alamos National Laboratory (LANL). The goal of this project is to develop a lightweight, semi-structural, radarabsorbing ceramic foam that can be incorporated into aircraft exhaust systems to replace many of the currently used dense ceramic parts and thereby improve the radar cross section. Although the conventional processes for producing ceramic foams have not been able to provide materials that meet the design specifications for high strength at low density, we have developed and demonstrated a novel sol-gel emulsion process for preparing microcellular ceramic foams in which compositional and microstructural control is expected to provide the requisite high-temperature radar-absorption, strength-to-weight ratio, and thermal insulative properties.
\end{abstract}

\section{Background and Research Objectives}

The exhaust systems of military jet engines must be constructed using radar absorbing ceramic materials that can withstand harsh thermal, chemical, and mechanical environments. Currently, these exhaust system structures combine relatively dense ceramic matrix composites (CMCs) and a lightweight, non-structural porous ceramic in a proprietary configuration that is heavy (compromising overall airframe design) and that provides inadequate control over both radar and infrared signatures. The short in-service lifetimes of these structures has prompted interest in designs having improved durability and containing fewer parts.

*Principal investigator, e-mail: huling@lanl.gov 
A viable approach for reducing both weight and radar cross section (RCS) is to replace the (non-structural) porous ceramic and (structural) ceramic matrix composite with a continuous layer of a lightweight, semi-structural, radar-absorbing ceramic foam, as illustrated in Figure 1a. This substitution creates a component having improved electrical and dimensional homogeneity, thus minimizing reflection and maximizing potential absorption.

The proposed use of ceramic foams as high-temperature, semi-structural radar-absorbing structure (RAS) is a direct analogue of the successful use of organic (carbon/polymer) foams as RAS in low-temperature, non-aggressive aerospace applications. In both cases, the foam microstructure allows the absorber to be lightweight and to have a surface impedance closely matched to that of free space, thus minimizing reflection from the front face. As a wave progresses through the material, however, a controlled increase in dielectric and/or magnetic losses (achieved during foam processing by the incorporation and graded distribution of radar absorbing material, RAM) dissipates the wave's electromagnetic energy. The load-bearing capacity of rigid foams allows dense, reflecting materials to be partially or entirely replaced, thus improving RCS characteristics.

Vital aspects of the problem, however, require that advanced ceramics be used as opposed to even the most heat-resistant polymers. The planned application involves periods of severe chemical corrosion and mechanical and thermal stress at temperatures up to $1800^{\circ} \mathrm{F}$, including intervals requiring radar absorption. Technology for control of RCS exists, having been developed using polymer foam materials, so we focus here on the problem of achieving

high temperature structural integrity of the foams (by using ceramic materials) and advancing the polymer foam RAS technology to achieve high temperature radar absorption.

The research objectives include: 1) minimizing mechanical and thermal expansion strain by utilizing a lightweight, refractory ceramic foam, 2) reducing the cell size of the foams by more than an order of magnitude compared to conventionally-produced ceramic foams to maximize the strength-to-weight ratio, and 3) minimizing the foam's thermal conductivity and chemical penetrability to keep dispersed magnetic RAM below its Curie temperature and provide enhanced protection of both the RAM and substructure.

\section{Importance to LANL's Science and Technology Base and National R\&D Needs}

By promoting the development of a "reduced signature technology," this project addresses the radar/IR detection of military aircraft-a problem of national interest. The development of new materials and processes for durable high-temperature RAS is a high priority shared by the "big three" defense aerospace contractors: existing components cannot be used above $1600^{\circ} \mathrm{F}$ and do not survive the desired 500 hours. Industry and the Department 
of Defense (DoD) are receptive to the foam approach because carbon/polymer foam RAS have already proven effective in less extreme aircraft environments. We are aware of no other concerted efforts to develop microcellular ceramic foams for this application. Indeed, the process technology developed for this project is exclusive to Los Alamos and represents the only known process for preparing filled (i.e., second-phase doped) ceramic foams having adequately low density and high-temperature $\left(>1800^{\circ} \mathrm{F}\right)$ strength and chemical resistance.

The importance of these microcellular ceramic foams to the Laboratory's science and technology base is not limited to RAS applications. These foams have exceptionally low densities ( $<10 \%$ theoretical) and fine microstructures ( $<10$ micron cell sizes). The small cell size and closed-cell morphology of the foams is predictive of superior strength-to-weight and thermal insulation properties. While these properties have been combined in polymer foams, in advanced ceramics having applications at high temperatures and in aggressive chemical environments (e.g., aerospace), such materials are unique. Vitreous silica foams can be prepared having relative densities as low as $3 \%$, consistent with the goal of producing "ultralow-density silica glass foams" stated in the 1992 Department of Energy (DOE) Defense Critical Technologies Plan. Numerous applications relevant to industry as well as defense and energy (improved thermal insulation, advanced fuel cells, fast-response sensors, low permitivity dielectric packaging) exist for potential future commercialization. Indeed, a recent article in Time magazine on 21st century "technologies that will change our lives" noted that "Styrofoam-like ceramic foams - virtually weightless but efficient at trapping heat-will take over from foam plastics wherever lightweight insulation is in demand" (July 17, 1995; p.39).

\section{Scientific Approach and Results to Date}

Although ceramics are the most suitable materials for withstanding the extreme conditions associated with jet engine exhaust, ceramic foams prepared by the well-established methods do not combine the strength-to-weight properties, thermal stability, and processing flexibility necessary to find application as high-temperature RAS. The strength of ceramic foams prepared by traditional approaches such as blowing or replication/burn-out of polymer templates is limited both by the porosity of the cell walls and the large cell (i.e., effective "flaw") size. We have developed and demonstrated a novel sol-gel / emulsion process (referred to here as the "solids-stabilized emulsion" process) for preparing microcellular ceramic foams having highly refined cell structures (currently $\sim 10$ microns) and thin, dense cell walls (Figure 1b). This approach both enhances the solids loading (and, therefore, strength) of the cell walls and reduces the cell size by more than an order of magnitude 
compared to traditional ceramic foams. Paradoxically, these denser walls give the emulsionderived foams comparable strength and lower permeability at lower overall densities $(<10 \%$ theoretical) than the more traditional foams. This technology will provide a stronger, lighter, better-insulating host for advanced high-temperature RAM as well as improved flexibility in the control of IR emission.

In FY95, we extended our process to the preparation of crystalline mullite microcellular foams (maximum use temperature $2800-3000^{\circ} \mathrm{F}$ ), from initial work using vitreous silica (maximum use temperature $1400-1600^{\circ} \mathrm{F}$ ). This is a nontrivial achievement, considering the added thermal, mechanical, and chemical stability of mullite and the added difficulty of synthesizing multi-component ceramics within the constraint of maintaining a lightweight foam microstructure. We are currently investigating the scale-up of the process, and RAM incorporation is expected to begin early in FY96 as part of an industrial collaboration with Northrop-Grumman.

a) Microcellular Mullite Foam Processing. Mullite $\left(3 \mathrm{Al}_{2} \mathrm{O}_{3} \cdot 2 \mathrm{SiO}_{2}\right)$ is the preferred material for RAS ceramic foams due to its maintenance of full strength at temperatures well above $1800^{\circ} \mathrm{F}$; its high resistance to creep; and its low thermal expansion coefficient, which provides high resistance to thermal shock. The theoretical density of mullite is lower than that of $\alpha$-alumina, its sol-gel processing has been extensively studied, and its chemical precursors are relatively inexpensive.

Mullite foam processing and scale-up (see below) were investigated in parallel to ensure that the final processing route would yield microcellular foams fulfilling both compositional (phase-pure mullite or mullite-alumina mixture) and dimensional (volumes $>250 \mathrm{~cm}^{3}$ ) requirements. Mullite contains much more alumina than silica, and while microcellular silica foams are easily prepared due to silica's controllable sol-gel chemistry, the reactivity of organometallic alumina precursors towards water prevents their controlled use in an emulsion process. Alternately, techniques involving diffusion of alumina precursors into an existing silica foam (prior to drying or firing) are not practical when the sample size exceeds several cubic centimeters. The preferred approach for making large samples minimizes the diffusion of liquids and gases through the foam structure during synthesis and heating. Colloidal $\gamma$ alumina is mixed with $5-10 \mu \mathrm{m}$ spherical silica "shells" dispersed in ethanol and derived from a solids-stabilized emulsion. Heterocoagulation of the alumina and silica by adjustment of $\mathrm{pH}$ ensures good mixing of the mullite precursors prior to consolidation and increases the integrity of the dried, unfired foam.

b) Scale-Up. Currently, a benchtop pressure filtration system consolidates the galumina/silica microsphere sols using applied gas pressure, which reduces the likelihood of sample cracking and RAM segregation, and produces samples large enough (12-cm diameter) 
for all property measurements of interest. [The foams' emulsion precursors were originally consolidated in a centrifuge. Centrifugation is adequate for process development using small samples, but scale-up and RAM incorporation using centrifugation are problematic due to severe limitations on sample size, the gradient in applied consolidation pressure along the height of the sample (which promotes sample cracking), and the tendency for dense second phases in the emulsion to settle rapidly and become segregated.] In contrast to early experiments in which the emulsions were consolidated prior to "shell formation" (i.e., the emulsion was initially centrifuged until the dispersed droplets formed a space-filling bi-liquid foam strengthened by sol-gel deposition from the liquid phase), pressure filtration after shell formation forms a less dense, more open foam microstructure that dries faster with less cracking. 


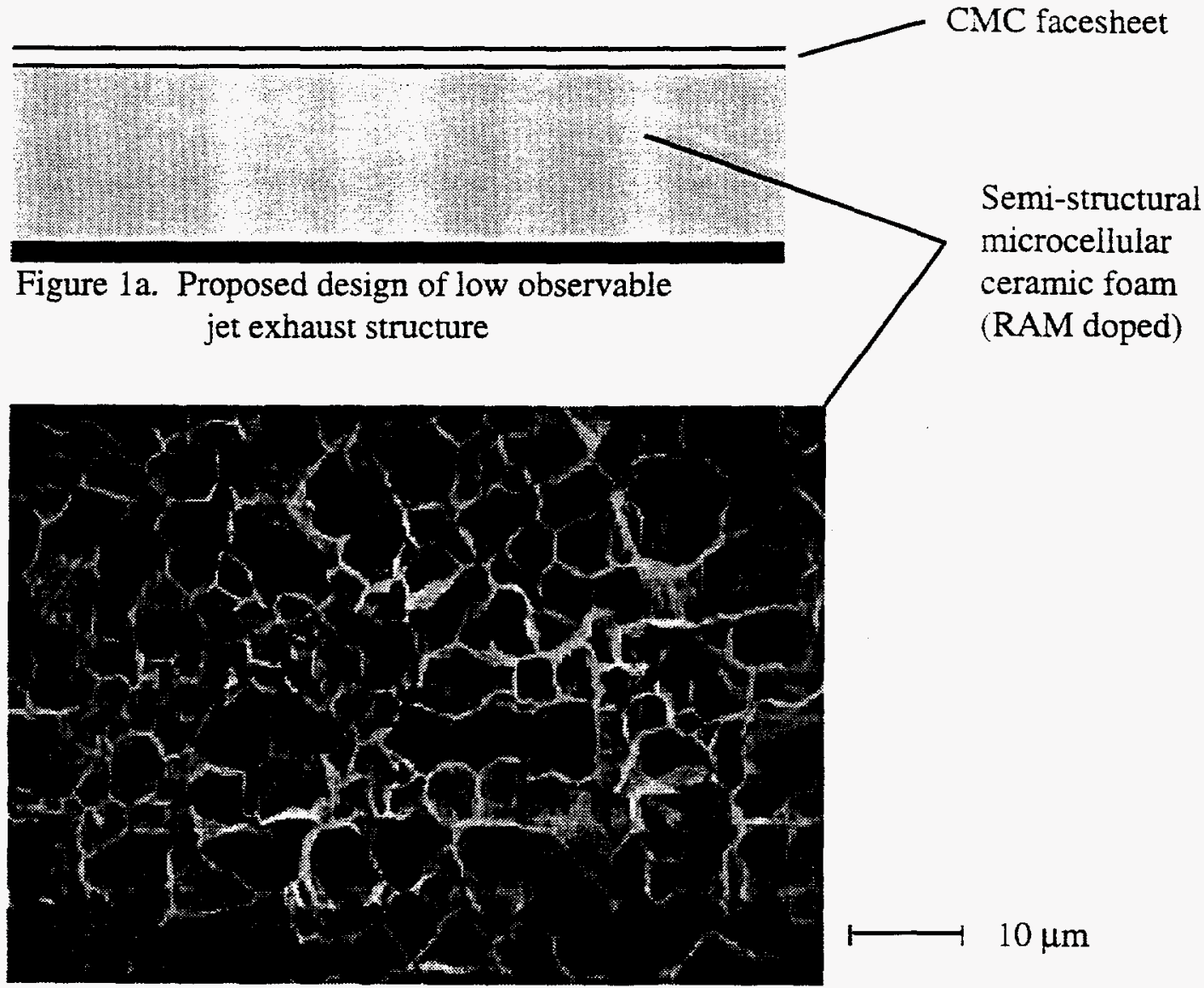

Figure $1 b$. Los Alamos microcellular mullite foam 